\title{
$\Upsilon(3 \mathrm{~S})$ and $\chi_{\mathrm{b}}(3 \mathrm{P})$ production and polarization in the NRQCD with $\mathrm{k}_{\mathrm{T}}$-factorization
}

\author{
Nizami Abdulov ${ }^{1, *}$ and Artem Lipatov $^{2,3}$ \\ ${ }^{1}$ Faculty of Physics, Lomonosov Moscow State University, 119991 Moscow, Russia \\ ${ }^{2}$ Skobeltsyn Institute of Nuclear Physics, Lomonosov Moscow State University, 119991 \\ Moscow, Russia \\ ${ }^{3}$ Joint Institute for Nuclear Research, 141980 Dubna, Moscow Region, Russia
}

\begin{abstract}
The $\Upsilon(3 S)$ production and polarization at high energies is studied in the framework of $k_{T}$-factorization approach. Our consideration is based on the non-relativistic QCD formalism for bound states formation and off-shell production amplitudes for hard partonic subprocesses. The transverse momentum dependent (TMD, or unintegrated) gluon densities in a proton were derived from the CiafaloniCatani-Fiorani-Marchesini (CCFM) evolution equation as well as from the Kimber-Martin-Ryskin (KMR) prescription. Treating the nonperturbative color octet transitions in terms of the mulitpole radiation theory and taking into account feed-down contributions from radiative $\chi_{b}(3 P)$ decays, we extract the corresponding non-perturbative matrix elements for $\Upsilon(3 S)$ and $\chi_{b}(3 P)$ mesons from a combined fit to $\Upsilon(3 S)$ transverse momenta distributions measured by the CMS and ATLAS Collaborations at the LHC energies $\sqrt{s}=7$ and $13 \mathrm{TeV}$ and central rapidities. Then we apply the extracted values to investigate the polarization parameters $\lambda_{\theta}, \lambda_{\phi}$ and $\lambda_{\theta \phi}$, which determine the $\Upsilon(3 S)$ spin density matrix. Our predictions have a good agreement with the currently available data within the theoretical and experimental uncertainties.
\end{abstract}

\section{Introduction}

In recent years, the production processes of quarkonia $J / \psi$ and $\Upsilon(n S)$ have been actively studied after the discovery of a strong discrepancy between theoretical predictions within the framework of the color singlet model (CS) and the data obtained at the Tevatron. A theoretical framework for the description of heavy quarkonia production and decays is provided by the non-relativistic QCD (NRQCD) factorization $[1,2]$. NRQCD is an effective field theory, which is used to describe the production of heavy quarkonia, based on the expansion in $v$ and $\alpha_{s}$ :

$$
\begin{aligned}
\left|\psi_{Q}\right\rangle=O(1)\left|Q \bar{Q}\left[{ }^{3} S_{1}^{(1)}\right]\right\rangle+ & O(v)\left|Q \bar{Q}\left[{ }^{3} P_{J}^{(8)}\right] g\right\rangle+O\left(v^{2}\right)\left|Q \bar{Q}\left[{ }^{3} S_{1}^{(1,8)}\right] g g\right\rangle+ \\
& +O\left(v^{2}\right)\left|Q \bar{Q}\left[{ }^{1} S_{0}^{(8)}\right] g\right\rangle+O\left(v^{2}\right)\left|Q \bar{Q}\left[{ }^{3} D_{J}^{(1,8)}\right] g g\right\rangle+\ldots
\end{aligned}
$$

\footnotetext{
*e-mail: nizami.abdulov@gmail.com
} 
This formalism implies a separation of perturbatively calculated short-distance cross-sections for the production of $Q \bar{Q}$ pair in an intermediate Fock state ${ }^{2 S+1} L_{J}^{(a)}$ and long-distance non-perturbative matrix elements (NMEs), which describe the transition of that intermediate $Q \bar{Q}$ state into a physical quarkonium via soft gluon radiation. They are assumed to be universal (process- and energy-independent), not dependent on the quarkonium momentum and obeying certain hierarchy in powers of the relative heavy quark velocity $v_{Q} \sim \log ^{-1} m_{Q} / \Lambda_{Q C D}[3]$. The color octet (CO) NMEs are not calculable within the theory and have to be only extracted from the data.

The cross sections of prompt $S$-and $P$-wave quarkonia production in $p p$ collisions are known at the NLO NRQCD and the dominant tree-level NNLO* corrections to the color-singlet production mechanism have been calculated [4]. With properly adjusted values of NMEs, one can achieve a good agreement between the NLO NRQCD predictions and the experimental data on the quarkonia transverse momenta distributions [5-9]. However, the extracted NMEs strongly depend on the minimal quarkonia transverse momentum $p_{T}$ used in the fits and are almost incompatible with each other when obtained from fitting different data sets. Moreover, none of the fits can simultaneously describe data on the polarization and the quarkonia production.

A possible solution to the "polarization puzzle" has been proposed recently in the framework of a model that interprets the soft final state gluon radiation as a series of color-electric dipole transitions [10]. It was already used for the prompt charmonia production [11]. In this approach, a soft gluon with a small energy $E \sim$ $\Lambda_{Q C D}$ is emitted after the hard interaction is over, bringing away the unwanted color and changing other quantum numbers of the produced CO system. Thus, having small energy of the emitted gluons gives us the confidence that we do not enter the confinement or perturbative domains. In our calculations such soft gluon emission is described by a classical multipole expansion, in which the electric dipole $(E 1)$ transition dominates. The proposed approach leads to unpolarized or only weakly polarized quarkonia either because of the cancellation between the ${ }^{3} P_{1}^{(8)}$ and ${ }^{3} P_{2}^{(8)}$ contributions or as a result of two successive color-electric $E 1$ dipole transitions in the chain ${ }^{3} S_{1}^{(8)} \rightarrow{ }^{3} P_{J}^{(8)} \rightarrow{ }^{3} S_{1}^{(1)}$ giving us the possibility to simultaneously describe the polarization parameters and production for mesons.

The main goal of this research is a detailed study of the bottomonia production processes and their polarization properties at the LHC. The data has been measured recently by the CMS $[12,13]$, ATLAS [14] and LHCb [15] Collaborations. And polarization of $\Upsilon(n S)$ mesons has been investigated by the CMS Collaboration [16]. Due to heavier masses of bottomonia and smaller relative velocity $v_{b}$ of $b$ quarks in the bottomonium rest frame $\left(v_{b} \simeq 0.08\right.$ against $\left.v_{c} \simeq 0.23\right)$, these processes could be even a more suitable case to apply because of a more faster convergence of the double NRQCD expansion in strong coupling $\alpha_{s}$ and $v_{Q}$.

We are taking into account the latest measurements on the $\chi_{b}(m P)$ production. The latter have been observed recently by the LHCb Collaboration [15] for the first time and found to be rather significant (up to 40\%). To describe the perturbative production of the $b \bar{b}$ pair, the $k_{T}$-factorization approach of QCD is used by taking into account both CS and CO contributions. This approach is based on the Balitsky-Fadin-Kuraev-Lipatov (BFKL) [17] or Ciafaloni-Catani-Fiorani-Marchesini (CCFM) [18] evolution equations, which resum large logarithmic terms proportional to $\ln s \sim \ln (1 / x)$, important at high energies. The $k_{T}$-factorization approach has certain technical advantages in the ease of including higher-order radiative correc- 
tions (namely, leading part of $\mathrm{NLO}+\mathrm{NNLO}+\ldots$ terms) in the form of transverse momentum dependent (TMD, or unintegrated) gluon density function in a proton $f\left(x, \mathbf{k}_{T}^{2}, \mu^{2}\right)$.

\section{The theoretical approach}

Our consideration is based on the off-shell gluon-gluon fusion subprocesses that represent the true $\mathrm{LO}$ in QCD:

$$
\begin{gathered}
g^{*}\left(k_{1}\right)+g^{*}\left(k_{2}\right) \rightarrow \Upsilon\left[{ }^{3} S_{1}^{(1)}\right](p)+g(k), \\
g^{*}\left(k_{1}\right)+g^{*}\left(k_{2}\right) \rightarrow \Upsilon\left[{ }^{1} S_{0}^{(8)},{ }^{3} S_{1}^{(8)},{ }^{3} P_{J}^{(8)}\right](p), \\
g^{*}\left(k_{1}\right)+g^{*}\left(k_{2}\right) \rightarrow \chi_{b J}(p)\left[{ }^{3} P_{J}^{(1)},{ }^{3} S_{1}^{(8)}\right] \rightarrow \Upsilon\left(p_{1}\right)+\gamma\left(p_{2}\right),
\end{gathered}
$$

where $J=0,1$ or 2 and the four-momenta of all particles are indicated in the parentheses. To obtain the production amplitudes for $b \bar{b}$ states with required quantum numbers from the ones for an unspecified $b \bar{b}$ state we use the appropriate projection operators, see [19].

To calculate off-shell production amplitudes we integrate the product of the hard scattering amplitude $A(q)$ expanded in a series around $q=0$ and meson bound state wave function $\Psi^{(a)}(q)$ with respect to $q$ :

$$
A(q) \Psi^{(a)}(q)=\left.A\right|_{q=0} \Psi^{(a)}(q)+\left.q^{\alpha}\left(\partial A / \partial q^{\alpha}\right)\right|_{q=0} \Psi^{(a)}(q)+\ldots
$$

A term-by-term integration of this series employs the identities:

$$
\begin{gathered}
\int \frac{d^{3} q}{(2 \pi)^{3}} \Psi^{(a)}(q)=\frac{1}{\sqrt{4 \pi}} \mathcal{R}^{(a)}(0), \\
\int \frac{d^{3} q}{(2 \pi)^{3}} q^{\alpha} \Psi^{(a)}(q)=-i \epsilon^{\alpha}\left(L_{z}\right) \frac{\sqrt{3}}{\sqrt{4 \pi}} \mathcal{R}^{\prime(a)}(0),
\end{gathered}
$$

where $\mathcal{R}^{(a)}(x)$ is the radial wave function in the coordinate representation.

The corresponding NMEs are directly related to the wave functions $\mathcal{R}^{(a)}(x)$ and their derivatives:

$$
\begin{aligned}
& \left\langle\mathcal{O}^{\mathcal{Q}}\left[{ }^{2 S+1} L_{J}^{(a)}\right]\right\rangle=2 N_{c}(2 J+1)\left|\mathcal{R}^{(a)}(0)\right|^{2} / 4 \pi, \\
& \left\langle\mathcal{O}^{\mathcal{Q}}\left[{ }^{2 S+1} L_{J}^{(a)}\right]\right\rangle=6 N_{c}(2 J+1)\left|\mathcal{R}^{\prime(a)}(0)\right|^{2} / 4 \pi
\end{aligned}
$$

for $S$ - and $P$-wave quarkonium $\mathcal{Q}$ respectively, where $N_{c}=3$.

The summation over polarizations of the incoming off-shell gluons is performed according the BFKL prescription $\sum \epsilon^{\mu} \epsilon^{* \nu}=\mathbf{k}_{T}^{\mu} \mathbf{k}_{T}^{\nu} / \mathbf{k}_{T}^{2}$. The spin density matrix of the $S$-wave quarkonia is expressed in terms of the momenta $l_{1}$ and $l_{2}$ of the decay leptons:

$$
\sum \epsilon^{\mu} \epsilon^{* \nu}=3\left(l_{1}^{\mu} l_{2}^{\nu}+l_{1}^{\nu} l_{2}^{\mu}-\frac{m^{2}}{2} g^{\mu \nu}\right) / m^{2}
$$

This expression is equivalent to the standard expression $\sum \epsilon^{\mu} \epsilon^{* \nu}=-g^{\mu \nu}+$ $p^{\mu} p^{\nu} / m^{2}$. In all other respects the evaluation follows the standard QCD Feynman rules. The obtained results have been explicitly tested for gauge invariance: we have observed their gauge invariance even with off-shell initial gluons. 
The cross sections of $\Upsilon(3 S)$ and $\chi_{b}(3 P)$ production in the $k_{T}$-factorization approach are calculated as a convolution of the off-shell partonic cross sections and TMD gluon densities in a proton:

$$
\begin{aligned}
\sigma=\int \frac{1}{8 \pi\left(x_{1} x_{2} s\right) F} f_{g}\left(x_{1}, \mathbf{k}_{1 T}^{2}, \mu^{2}\right) f_{g}\left(x_{2}, \mathbf{k}_{2 T}^{2}, \mu^{2}\right) \times \\
\times \overline{\left|A\left(g^{*}+g^{*} \rightarrow Q \bar{Q}+g\right)\right|^{2}} d \mathbf{p}_{T}^{2} d \mathbf{k}_{1 T}^{2} d \mathbf{k}_{2 T}^{2} d y d y_{g} \frac{d \phi_{1}}{2 \pi} \frac{d \phi_{2}}{2 \pi}, \\
\sigma=\int \frac{2 \pi}{x_{1} x_{2} s F} f_{g}\left(x_{1}, \mathbf{k}_{1 T}^{2}, \mu^{2}\right) f_{g}\left(x_{2}, \mathbf{k}_{2 T}^{2}, \mu^{2}\right) \times \\
\times \overline{\left|A\left(g^{*}+g^{*} \rightarrow Q \bar{Q}\right)\right|^{2}} d \mathbf{k}_{1 T}^{2} d \mathbf{k}_{2 T}^{2} d y \frac{d \phi_{1}}{2 \pi} \frac{d \phi_{2}}{2 \pi} .
\end{aligned}
$$

where $\phi_{1}$ and $\phi_{2}$ are the azimuthal angles of the initial off-shell gluons having the fraction of the momentum $x_{1}$ and $x_{2}$ and non-zero transverse momenta $\mathbf{k}_{1 T}^{2}$ and $\mathbf{k}_{2 T}^{2}$, $\mathbf{p}_{T}^{2}$ and $y$ are the transverse momentum and rapidity of produced mesons, $y_{g}$ is the rapidity of outgoing gluon and $\sqrt{s}$ is the $p p$ center-of-mass energy, the off-shell gluon flux factor $F$ is defined as $F=2 \lambda^{1 / 2}\left(\hat{s}, k_{1}^{2}, k_{2}^{2}\right)$, where $\hat{s}=\left(k_{1}+k_{2}\right)^{2}$ and $\lambda(x, y, z)$ is the known kinematic function.

\section{Results}

In this paper we use several TMD gluon distribution functions to describe the cross sections of the inclusive production $\Upsilon(3 S)$ : A0 [20], JH'2013 set 1 [21] and KMR [22]. As a commonly adopted choice, we set CS NMEs $\left\langle\mathcal{O}\left(\Upsilon\left[{ }^{3} S_{1}^{(1)}\right]\right)\right\rangle=3.54$ $\mathrm{GeV}^{3}$ and $\left\langle\mathcal{O}\left(\chi\left[{ }^{3} P_{0}^{(1)}\right]\right)\right\rangle=2.83 \mathrm{GeV}^{5}$. These values were obtained in the potential model calculations [23]. For more information about parameters and values, which are used in this study, see [19].

We have performed a global fit to the $\Upsilon(3 S)$ production data at the LHC and determined the corresponding NMEs for both $\Upsilon(3 S)$ and $\chi_{b}(3 P)$ mesons. We have included in the fitting procedure the $\Upsilon(3 S)$ transverse momentum distributions measured by the CMS $[12,13]$ and ATLAS [14] Collaborations at $\sqrt{s}=7$ and $13 \mathrm{TeV}$ and central rapidities, where our $k_{T}$-factorization calculations are most relevant due to essentially low- $x$ region probed. We have excluded from our fit low $p_{T}$ region and consider only the data at $p_{T}>p_{T}^{\text {cut }}=10 \mathrm{GeV}$, where the NRQCD formalism is believed to be most reliable. To determine NMEs for $\chi_{b}(3 P)$ mesons, we also included into the fit the recent $\mathrm{LHCb}$ data [15] on the radiative $\chi_{b}(3 P) \rightarrow \Upsilon(3 S)+\gamma$ decays taken at $\sqrt{s}=7$ and $8 \mathrm{TeV}$. We found that the $p_{T}$ shape of the direct $\Upsilon\left[{ }^{3} S_{1}^{(8)}\right]$ and feed-down $\chi_{b}\left[{ }^{3} S_{1}^{(8)}\right]$ contributions is almost the same in all kinematical regions probed by the LHC and Tevatron experiments, i.e. the ratio

$$
r=\frac{\sum_{J=0}^{2}(2 J+1) B\left(\chi_{b J}(3 P) \rightarrow \Upsilon(3 S)+\gamma\right) d \sigma\left[\chi_{b J}(3 P),{ }^{3} S_{1}^{(8)}\right] / d p_{T}}{d \sigma\left[\Upsilon(3 S),{ }^{3} S_{1}^{(8)}\right] / d p_{T}}
$$

can be well approximated by a constant for a wide $\Upsilon(3 S)$ transverse momentum $p_{T}$ and rapidity $y$ ranges at different energies. We estimate the mean-square average 
$r=0.654 \pm 0.005$, which is practically independent on the TMD gluon density in a proton.

Since up to now there are no experimental data on the $\chi_{b}(3 P)$ transverse momentum distributions, we cannot separately determine the values of $\left\langle\mathcal{O}^{\Upsilon(3 S)}\left[{ }^{3} S_{1}^{(8)}\right]\right\rangle$ and $\left\langle\mathcal{O}^{\chi_{b 0}(3 P)}\left[{ }^{3} S_{1}^{(8)}\right]\right\rangle$ from the available $\Upsilon(3 S)$ data. Instead, we introduce the linear combination

$$
M_{r}=\left\langle\mathcal{O}^{\Upsilon(3 S)}\left[{ }^{3} S_{1}^{(8)}\right]\right\rangle+r\left\langle\mathcal{O}^{\chi} \chi_{b 0}(3 P)\left[{ }^{3} S_{1}^{(8)}\right]\right\rangle,
$$

which can be extracted from the measured $\Upsilon(3 S)$ transverse momentum distributions.

Then we use recent $\mathrm{LHCb}$ data [15] on the fraction of $\Upsilon(3 S)$ mesons originating from the $\chi_{b}(3 P)$ radiative decays measured at $\sqrt{s}=7$ and $8 \mathrm{TeV}$. The $\mathrm{LHCb}$ Collaboration reported the ratio:

$$
R_{\Upsilon(3 S)}^{\chi_{b}(3 P)}=\sum_{J=1}^{2} \frac{\sigma\left(p p \rightarrow \chi_{b J}(3 P)+X\right)}{\sigma(p p \rightarrow \Upsilon(3 S)+X)} \times B\left(\chi_{b J} \rightarrow \Upsilon(3 S)+\gamma\right)
$$

From the known $M_{r}$ and $R_{\Upsilon(3 S)}^{\chi_{b}(3 P)}$ values one can separately determine the $\left\langle\mathcal{O}^{\Upsilon(3 S)}\left[{ }^{3} S_{1}^{(8)}\right]\right\rangle$ and $\left\langle\mathcal{O}^{\chi_{b 0}(3 P)}\left[{ }^{3} S_{1}^{(8)}\right]\right\rangle$, thus reconstructing full map of color octet NMEs for both $\Upsilon(3 S)$ and $\chi_{b}(3 P)$ mesons. The results of our fits are collected in Table 1 . For comparison, we also presented there the NMEs obtained in the conventional NLO NRQCD by other authors [24].

Some of the results obtained in the fits are compared with our predictions in Figs. $1-2$ (for others, see [19]). The shaded areas represent the theoretical uncertainties of our calculations, which include the scale uncertainties, uncertainties coming from the NME fitting procedure and uncertainties connected with the choice of the intermediate color-octet mass, added in quadrature.

As it is well known, the polarization of any vector meson can be described with three parameters $\lambda_{\theta}, \lambda_{\phi}$ and $\lambda_{\theta \phi}$, which determine the spin density matrix of a meson decaying into a lepton pair and can be measured experimentally. The double differential angular distribution of the decay leptons can be written as [25]:

$$
\frac{d \sigma}{d \cos \theta^{*} d \phi^{*}} \sim \frac{1}{3+\lambda_{\theta}}\left(1+\lambda_{\theta} \cos ^{2} \theta^{*}+\lambda_{\phi} \sin ^{2} \theta^{*} \cos 2 \phi^{*}+\lambda_{\theta \phi} \sin 2 \theta^{*} \cos \phi^{*}\right),
$$

where $\theta^{*}$ and $\phi^{*}$ are the polar and azimuthal angles of the decay lepton measured in the meson rest frame. One of the result for the experimental data from CMS [16] in the Collins-Soper frame is presented in Fig. 3, including the frame-independent parameter $\tilde{\lambda}=\left(\lambda_{\theta}+3 \lambda_{\phi}\right) /\left(1-\lambda_{\phi}\right)$. These calculations were done using the A0 gluon density which provides a best description of the measured $\Upsilon(3 S)$ transverse momenta distributions.

\section{Conclusion}

We have considered the $\Upsilon(3 S)$ production at the LHC in the framework of $k_{T^{-}}$ factorization approach. Our consideration was based on the off-shell production amplitudes for hard partonic subprocesses (including both color-singlet and color-octet contributions), NRQCD formalism for the formation of bound states and TMD gluon densities in a proton (derived from the CCFM evolution equation and KMR scheme as well). Treating the nonperturbative color octet transitions in terms of multipole radiation theory and taking into account feed-down contributions from the radiative 
$\chi_{b}(3 P)$ decays, we extracted $\Upsilon(3 S)$ and $\chi_{b}(3 P)$ NMEs in a fit to $\Upsilon(3 S)$ transverse momentum distributions measured by the CMS and ATLAS Collaborations at $\sqrt{s}=7$ and $13 \mathrm{TeV}$. We have inspected the extracted NMEs with the available LHC data taken in the different kinematical regions and demostrated that these NMEs do not contradict the data. Then we estimated polarization parameters $\lambda_{\theta}, \lambda_{\phi}, \lambda_{\theta \phi}$ and frame-independent parameter $\tilde{\lambda}$ which determine the $\Upsilon(3 S)$ spin density matrix. We show that treating the soft gluon emission as a series of explicit color-electric dipole transitions within the NRQCD leads to unpolarized $\Upsilon(3 S)$ production at moderate and large transverse momenta, that is in agreement with the LHC data.

\section{Acknowledgements}

The authors thank S.P. Baranov, M.A. Malyshev and H. Jung for their interest, useful discussions and important remarks. N.A.A. is supported by the Foundation for the Advancement of Theoretical Physics and Mathematics "Basis" (Grant No. 18-15-33-1) and by the RFBR, project number 19-32-90096. A.V.L. is grateful the DESY Directorate for the support in the framework of Cooperation Agreement between MSU and DESY on phenomenology of the LHC processes and TMD parton densities.

\section{References}

[1] G. Bodwin, E. Braaten, G. Lepage, Phys. Rev. D 51, 1125 (1995).

[2] P. Cho, A.K. Leibovich, Phys. Rev. D 53, 150 (1996); Phys. Rev. D 53, 6203 (1996).

[3] K.-T. Chao, Y.-Q. Ma, H.-S. Shao, K. Wang, Y.-J. Zhang, Phys. Rev. Lett. 108, 242004 (2012);

B. Gong, L.-P. Wan, J.-X. Wang, H.-F. Zhang, Phys. Rev. Lett. 110, 042002 (2013).

[4] P. Artoisenet et al., Phys. Rev. Lett. 101, 152001 (2008).

[5] B. Gong, X.Q. Li, J.-X. Wang, Phys. Lett. B 673, 197 (2009).

[6] Y.-Q. Ma, K. Wang, K.-T. Chao, Phys. Rev. Lett. 106, 042002 (2011).

[7] M. Butenschön, B.A. Kniehl, Phys. Rev. Lett. 108, 172002 (2012).

[8] K.-T. Chao, Y.-Q. Ma, H.-S. Shao, K. Wang, Y.-J. Zhang, Phys. Rev. Lett. 108, 242004 (2012).

[9] B. Gong, L.-P. Wan, J.-X. Wang, H.-F. Zhang, Phys. Rev. Lett. 110, 042002 (2013).

[10] S.P. Baranov, Phys. Rev. D 93, 054037 (2016).

[11] S.P. Baranov, A.V. Lipatov, Eur. Phys. J. C 79, 621 (2019);

S.P. Baranov, A.V. Lipatov, arXiv:1906.07182 [hep-ph].

[12] CMS Collaboration, Phys. Lett. B 749, 14 (2015).

[13] CMS Collaboration, Phys. Lett. B 780, 251 (2018).

[14] ATLAS Collaboration, Phys. Rev. D 87, 052004 (2013).

[15] LHCb Collaboration, Eur. Phys. J. C 74, 3092 (2014).

[16] CMS Collaboration, Phys. Rev. Lett. 110, 081802 (2013).

[17] E.A. Kuraev, L.N. Lipatov, V.S. Fadin, Sov. Phys. JETP 44, 443 (1976);

E.A. Kuraev, L.N. Lipatov, V.S. Fadin, Sov. Phys. JETP 45, 199 (1977);

I.I. Balitsky, L.N. Lipatov, Sov. J. Nucl. Phys. 28, 822 (1978).

[18] M. Ciafaloni, Nucl. Phys. B 296, 49 (1988);

S. Catani, F. Fiorani, G. Marchesini, Phys. Lett. B 234, 339 (1990); 
S. Catani, F. Fiorani, G. Marchesini, Nucl. Phys. B 336, 18 (1990);

G. Marchesini, Nucl. Phys. B 445, 49 (1995).

[19] N.A. Abdulov, A.V. Lipatov, Eur. Phys. J. C 79, 830 (2019).

[20] H. Jung, arXiv:hep-ph/0411287.

[21] F. Hautmann, H. Jung, Nucl. Phys. B 883, 1 (2014).

[22] M.A. Kimber, A.D. Martin, M.G. Ryskin, Phys. Rev. D 63, 114027 (2001);

A.D. Martin, M.G. Ryskin, G. Watt, Eur. Phys. J. C 31, 73 (2003);

A.D. Martin, M.G. Ryskin, G. Watt, Eur. Phys. J. C 66, 163 (2010).

[23] E.J. Eichten, C. Quigg, arXiv:1904.11542 [hep-ph].

[24] Y. Feng, B. Gong, L.-P. Wan, J.-X. Wang, H.-F. Zhang, Chin. Phys. C 39, 123102 (2015).

[25] M. Beneke, M. Krämer, M. Vänttinen, Phys. Rev. D 57, 4258 (1998).

\begin{tabular}{lcccc}
\hline \hline & A0 & JH'2013 set 1 & KMR & NLO NRQCD \\
\hline$\left\langle\mathcal{O}^{\Upsilon(3 S)}\left[{ }^{3} S_{1}^{(1)}\right]\right\rangle / \mathrm{GeV}^{3}$ & 3.54 & 3.54 & 3.54 & 3.54 \\
$\left\langle\mathcal{O}^{\Upsilon(3 S)}\left[{ }^{1} S_{0}^{(8)}\right]\right\rangle / \mathrm{GeV}^{3}$ & 0.0 & 0.0 & 0.0 & $-0.0107 \pm 0.0107$ \\
$\left\langle\mathcal{O}^{\Upsilon(3 S)}\left[{ }^{3} S_{1}^{(8)}\right]\right\rangle / \mathrm{GeV}^{3}$ & $0.018 \pm 0.001$ & $0.007 \pm 0.002$ & $0.006 \pm 0.001$ & $0.0271 \pm 0.0013$ \\
$\left\langle\mathcal{O}^{\Upsilon(3 S)}\left[{ }^{3} P_{0}^{(8)}\right]\right\rangle / \mathrm{GeV}^{5}$ & 0.0 & $0.09 \pm 0.03$ & $0.073 \pm 0.006$ & $0.0039 \pm 0.0023$ \\
$\left\langle\mathcal{O}^{\chi}{ }_{b 0}(3 P)\left[{ }^{3} P_{0}^{(1)}\right]\right\rangle / \mathrm{GeV}^{5}$ & 2.83 & 2.83 & 2.83 & 2.83 \\
$\left\langle\mathcal{O}^{\chi}{ }_{b 0}(3 P)\left[{ }^{3} S_{1}^{(8)}\right]\right\rangle / \mathrm{GeV}^{3}$ & $0.016 \pm 0.003$ & $0.009 \pm 0.001$ & $0.005 \pm 0.001$ & - \\
\hline \hline
\end{tabular}

Table 1. The NMEs for $\Upsilon(3 S)$ and $\chi_{b}(3 P)$ mesons as determined from our fit at $p_{T}^{\text {cut }}=10 \mathrm{GeV}$. The NMEs obtained in the NLO NRQCD [24] are shown for comparison.
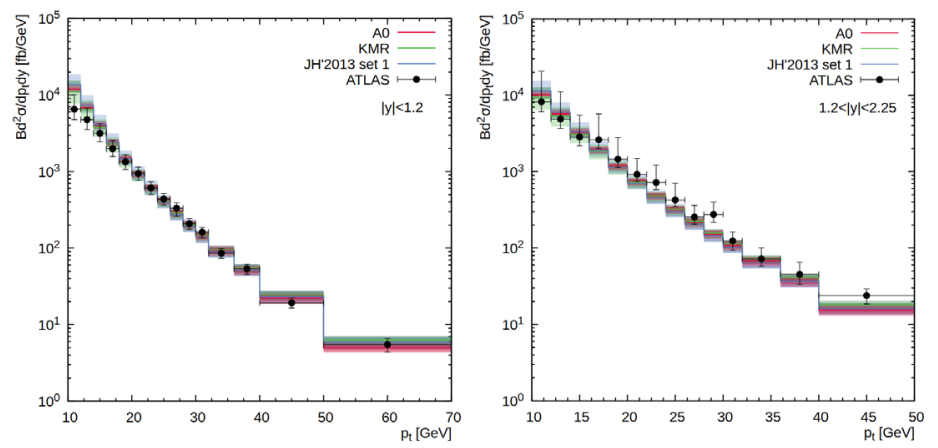

Figure 1. Transverse momentum distribution of inclusive $\Upsilon(3 S)$ production calculated at $\sqrt{s}=7 \mathrm{TeV}$ in the different rapidity regions. The red, green and blue histograms correspond to the predictions obtained with A0, KMR and JH'2013 set 1 gluon densities. Shaded bands represent the total uncertainties of our calculations, as it is described in text. The experimental data are from ATLAS [14]. 

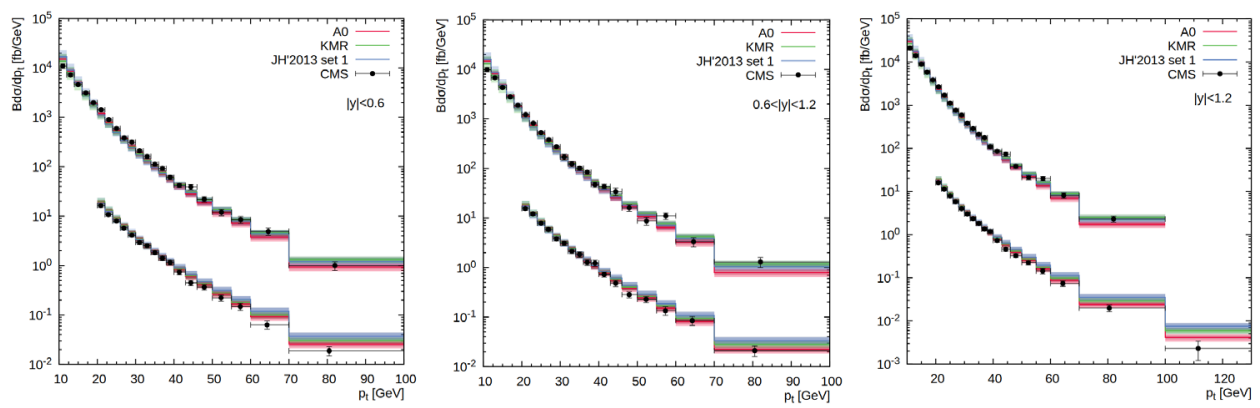

Figure 2. Transverse momentum distribution of inclusive $\Upsilon(3 S)$ production calculated at $\sqrt{s}=7 \mathrm{TeV}$ (upper histograms) and $\sqrt{s}=13 \mathrm{TeV}$ (lower histograms, divided by 100) in the different rapidity regions. Notation of all histograms is the same as in Fig. 1 . The experimental data are from CMS $[12,13]$.
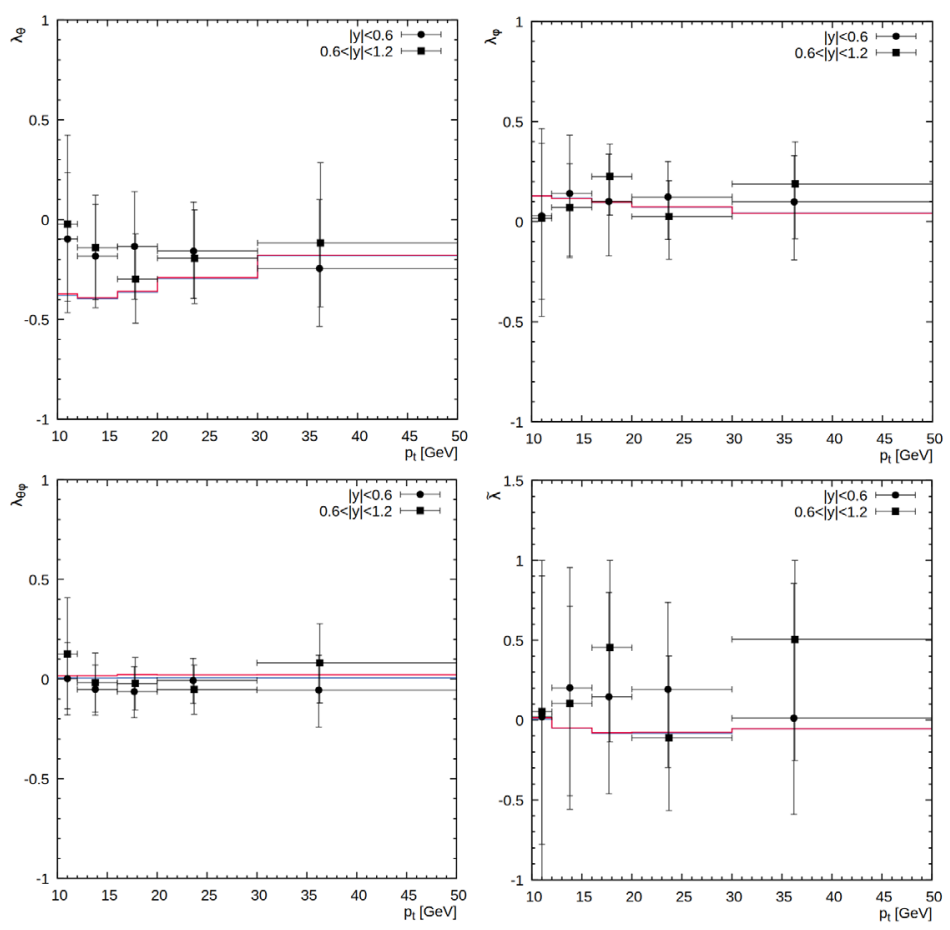

Figure 3. The polarization parameters $\lambda_{\theta}, \lambda_{\phi}, \lambda_{\theta \phi}$ and $\tilde{\lambda}$ of $\Upsilon(3 S)$ mesons calculated in the CS frame as function of its transverse momentum at $\sqrt{s}=7 \mathrm{TeV}$. The A0 gluon density is used. The blue and red histograms correspond to the predictions obtained at $|y|<0.6$ and $0.6<|y|<1.2$, respectively. The experimental data are from CMS [16]. 